\title{
Automorphism groups of some AG codes
}

\author{
David Joyner and Amy Ksir*
}

$1-19-2005$

\begin{abstract}
We show that in many cases, the automorphism group of a curve and the permutation automorphism group of a corresponding AG code are the same. This generalizes a result of Wesemeyer [W] beyond the case of planar curves.
\end{abstract}

\section{Introduction}

The construction of AG codes uses the Riemann-Roch space $L(D)$ associated to a divisor $D$ of a curve $X$ defined over a finite field [G]. Typically $X$ has no non-trivial automorphisms, but when it does we may ask how this can be used to better understand AG codes constructed from $X$.

Conversely, we may ask how the permutation automorphism group of an AG code corresponds with the automorphism group of the curve used to construct the code. In this paper we show that, in many cases, the automorphism group of a curve and the permutation automorphism group of a corresponding AG code are in fact the same.

Knowledge of which codes have large automorphism group can have applications to encoding (see [HLS]) and to decoding (indeed, permutation decoding has been implemented in version 2.0 of the error-correcting computer algebra package [GUAVA]).

*Mathematics Dept, USNA, Annapolis, MD 21402,wdj@usna.edu and ksir@usna.edu 


\section{The Riemann-Roch space $L(D)$ and the as- sociated AG code.}

Let $X$ be a smooth projective curve (scheme of dimension 1) over a finite field $F$, and let $F(X)$ denote the field of rational functions on $X$. If $D$ is any divisor on $X$, the Riemann-Roch space $L(D)$ is a finite dimensional $F$-vector space given by

$$
L(D)=L_{X}(D)=\left\{f \in F(X)^{\times} \mid \operatorname{div}(f)+D \geq 0\right\} \cup\{0\},
$$

where $\operatorname{div}(f)$ denotes the (principal) divisor of the function $f \in F(X)$. If $\bar{D}$ denotes the corresponding divisor over the algebraic closure $\bar{F}$, then $L(\bar{D})=$ $L(D) \otimes \bar{F}[$ Sti],$[\mathrm{TV}]$.

Let $P_{1}, \ldots, P_{n} \in X(F)$ be distinct points, and let $E=P_{1}+\ldots+P_{n}$ be the associated divisor. Let $D$ be a divisor of positive degree on $X$ such that $D$ and $E$ have disjoint support. Let $C=C(D, E)$ denote the $\mathrm{AG}$ code

$$
C=\left\{\left(f\left(P_{1}\right), \ldots, f\left(P_{n}\right)\right) \mid f \in L(D)\right\} .
$$

This is the image of $L(D)$ under the evaluation map

$$
\begin{gathered}
\operatorname{eval}_{E}: L(D) \rightarrow F^{n}, \\
f \longmapsto\left(f\left(P_{1}\right), \ldots, f\left(P_{n}\right)\right) .
\end{gathered}
$$

The kernel of the map $e^{2} a l_{E}$ is contained in $L(D-E)$, which is empty if $n>\operatorname{deg}(D)$. Thus for $n>\operatorname{deg}(D)$, eval $_{E}$ defines an isomorphism between $L(D)$ and the code $C(D, E)$.

\section{From curve automorphisms to code auto- morphisms.}

Now let $G$ be a group of automorphisms of the curve $X$, and assume that $D$ and $E$ are both stabilized by $G$. We will say that $G \subseteq \operatorname{Aut}_{D, E}(X)$. Then $G$ also acts on the code $C$, as follows.

The action of $\operatorname{Aut}(X)$ on $F(X)$ is defined as:

$$
\begin{array}{cl}
\operatorname{Aut}(X) & \longrightarrow \operatorname{Aut}(F(X)), \\
T & \longmapsto\left(f \longmapsto T^{*} f\right)
\end{array}
$$


where for any $P \in X, T^{*} f(P)=f\left(T^{-1}(P)\right.$ ). (We use $T^{-1}$ rather than $T$ here, to conform to the convention that the action should be on the left.)

Note that $Y=X / G$ is also smooth and $F(X)^{G}=F(Y)$.

Of course, $\operatorname{Aut}(X)$ also acts on the group $\operatorname{Div}(X)$ of divisors of $X$, denoted $T\left(\sum_{P} d_{P} P\right)=\sum_{P} d_{P} T(P)$, for $T \in \operatorname{Aut}(X), P$ a prime divisor, and $d_{P} \in \mathbb{Z}$. It is easy to see that $\operatorname{div}\left(T^{*} f\right)=T^{-1}(\operatorname{div}(f))$. Because of this, if $\operatorname{div}(f)+D \geq 0$ then $\operatorname{div}\left(T^{*} f\right)+T^{-1}(D) \geq 0$, for all $T \in \operatorname{Aut}(X)$. In particular, if the action of $G \subset \operatorname{Aut}(X)$ on $X$ leaves $D \in \operatorname{Div}(X)$ stable then $G$ also acts on $L(D)$. Assuming that $n>\operatorname{deg} D$, the isomorphism eval $_{E}: L(D) \rightarrow C$ will send this action to an action of $G$ on $C$. Specifically, each $T \in G$ acts by

$$
\begin{aligned}
\left(f\left(P_{1}\right), f\left(P_{2}\right), \ldots, f\left(P_{n}\right)\right) & \mapsto\left(T^{*} f\left(P_{1}\right), T^{*} f\left(P_{2}\right), \ldots, T^{*} f\left(P_{n}\right)\right) \\
& =\left(f\left(T^{-1}\left(P_{1}\right)\right), f\left(T^{-1}\left(P_{2}\right)\right), \ldots, f\left(T^{-1}\left(P_{n}\right)\right)\right) .
\end{aligned}
$$

If we also assume that $G$ leaves $E$ stable, then $G$ acts by permutations on the set $\left\{P_{1}, \ldots, P_{n}\right\}$. Thus $\left(T^{-1}\left(P_{1}\right), T^{-1}\left(P_{2}\right), \ldots, T^{-1}\left(P_{n}\right)\right)$ is a permutation

of the points $\left(P_{1}, P_{2}, \ldots, P_{n}\right)$, and the above action on $C$ simply permutes the corresponding coordinates.

Definition 1 The permutation automorphism group Perm $C$ of the code $C \subset F^{n}$ is the subgroup of $S_{n}$ (acting on $F^{n}$ by coordinate permutation) which preserves $C$.

Thus if $n>\operatorname{deg} D$, we have defined a homomorphism from $\operatorname{Aut}_{D, E}(X)$ to Perm $C$ :

$$
\begin{aligned}
\rho: \operatorname{Aut}_{D, E}(X) & \rightarrow \operatorname{Perm}_{C} . \\
T & \mapsto \operatorname{eval}_{E} \circ T^{*} \circ \operatorname{eval}_{E}^{-1}
\end{aligned}
$$

In the next section, we will construct an inverse for this homomorphism.

\section{From code automorphisms to curve auto- morphisms.}

Now we would like to answer the question: when does a group of permutation automorphisms of the code $C$ induce a group of automorphisms of the curve 
$X$ ? We will show that permutation automorphisms of the code $C(D, E)$ induce curve automorphisms whenever $D$ is very ample and the degree of $E$ is large enough. Under these conditions, the groups $\operatorname{Aut}_{D, E}(X)$ and Perm $C$ are isomorphic. In proving these facts, we generalize some results of Wesemeyer [W], who dealt with the planar case.

Theorem 2 Let $X$ be an algebraic curve, $D$ be a very ample divisor on $X$, and $P_{1} \ldots P_{n}$ be a set of points on $X$ disjoint from the support of $D$. Let $E=P_{1}+\ldots+P_{n}$ be the associated divisor, and $C=C(D, E)$ the associated $A G$ code. Let $G$ be the group of permutation automorphisms of $C$. Then there is an integer $r \geq 1$ such that if $n>r \cdot \operatorname{deg}(D)$, then $G$ can be lifted to a group of automorphisms of the curve $X$ itself. This lifting defines a group homomorphism $\psi: \operatorname{Perm} C \rightarrow \operatorname{Aut}(X)$. Furthermore, the lifted automorphisms will preserve $D$ and $E$, so the image of $\psi$ will be contained in $\operatorname{Aut}_{D, E}(X)$.

proof: First, note that since $n>\operatorname{deg} D$, eval $_{E}: L(D) \rightarrow C$ is a vector space isomorphism. Thus the permutation action of $G$ on $C$ can be pulled back to a linear action on $L(D)$. Next, we use $D$ to embed $X$ into projective space $\mathbb{P}^{d}$, where $d=\operatorname{dim} L(D)-1$. If we let $Y_{0}, \ldots, Y_{d}$ be a basis for $L(D)$, then the embedding is given explicitly by

$$
\begin{gathered}
\phi: X \rightarrow \mathbb{P}^{d}, \\
P \longmapsto\left[Y_{0}(P): \ldots: Y_{d}(P)\right] .
\end{gathered}
$$

The vector space action of $G$ on $L(D)$ induces an action on the polynomial ring $F\left[Y_{0}, \ldots, Y_{d}\right]$ and a projective linear action on $\mathbb{P}^{d}$. We will show that under the stated hypotheses, this action preserves the image of $X$ in $\mathbb{P}^{d}$, so restricts to an action on $X$. Furthermore, this action will stabilize the divisors $D$ and $E$.

To prove these claims, let us look more carefully at the action of $G$. Let $\tau$ be an element of $G$; it acts by a permutation of the coordinates of a point in $C$. The pullback of $\tau$ to $L(D)$ is the composition $e v a l_{E}^{-1} \circ \tau \circ e^{2} v a l_{E}$, which by abuse of notation we denote again by $\tau$. In the middle, $\tau$ acts as

$$
\left(f\left(P_{1}\right), \ldots, f\left(P_{n}\right)\right) \mapsto\left(f\left(P_{\tau(1)}\right), \ldots, f\left(P_{\tau(n)}\right)\right)
$$


where $f$ was a function in $L(D)$. Because the permutation action leaves the code $C$ invariant, this new point is also in the code. Since eval $_{E}$ is an isomorphism, there is a function which we will call $\tau(f)$ in $L(D)$ such that

$$
\left(f\left(P_{\tau(1)}\right), \ldots, f\left(P_{\tau(n)}\right)\right)=\left(\tau(f)\left(P_{1}\right), \ldots, \tau(f)\left(P_{n}\right)\right) .
$$

This defines the action of $G$ on $L(D)$. In particular, the action of $G$ on the basis $Y_{0}, \ldots, Y_{d}$ of $L(D)$ defines an action of $G$ on polynomial ring $F\left[Y_{0}, \ldots, Y_{d}\right]$ :

$$
Y_{0}^{e_{0}} \ldots Y_{d}^{e_{d}} \mapsto\left(\tau Y_{0}\right)^{e_{0}} \ldots\left(\tau Y_{d}\right)^{e_{d}}
$$

Then the action on the projective space $\mathbb{P}^{d}$ is as follows: an element $\tau$ of $G$ will act on a point $\left[Y_{0}: \ldots: Y_{d}\right]$ in $\mathbb{P}^{d}$ via

$$
\tau\left[Y_{0}: \ldots: Y_{d}\right]=\left[\tau^{-1} Y_{0}: \ldots: \tau^{-1} Y_{d}\right] .
$$

Now we will consider how this action on the projective space affects the images of the points $P_{1}, \ldots, P_{n}$ under the embedding $\phi$. For each point $P_{i}$, its image $\phi\left(P_{i}\right)$ has projective coordinates $\left[Y_{0}\left(P_{i}\right): \ldots: Y_{d}\left(P_{i}\right)\right]$. Then

$$
\begin{aligned}
\tau^{-1}\left(\phi\left(P_{i}\right)\right) & =\tau^{-1}\left[Y_{0}\left(P_{i}\right): \ldots: Y_{d}\left(P_{i}\right)\right] \\
& =\left[\tau Y_{0}\left(P_{i}\right): \ldots: \tau Y_{d}\left(P_{i}\right)\right] \\
& =\left[Y_{0}\left(P_{\tau(i)}\right): \ldots: Y_{d}\left(P_{\tau(i)}\right)\right] \\
& =\phi\left(P_{\tau(i)}\right) .
\end{aligned}
$$

Thus, a permutation of the code acts by the inverse permutation on the images of the points of $E$.

Now we would like to show that the image $\phi(X)$ is preserved by the action (6) of $G$ on $\mathbb{P}^{d}$. In the case where $d=1, X$ must have genus 0 and $\phi$ is an isomorphism, so this automatically holds. For $d>1$, the coordinates $Y_{i}$ must satisfy some homogeneous polynomial relations defining $\phi(X)$. Let $R_{1}\left(Y_{0}, \ldots, Y_{d}\right)=0, \ldots, R_{k}\left(Y_{0}, \ldots, Y_{d}\right)=0$ denote a set of polynomials of minimal degree that define the ideal of $\phi(X)$ in $\mathbb{P}^{d}$, so that its projective coordinate ring is

$$
F\left[Y_{0}, \ldots, Y_{d}\right] /\left(R_{1}, \ldots, R_{k}\right) .
$$

Since $R_{1}, \ldots, R_{k}$ are polynomials in $Y_{0}, \ldots, Y_{d}$, and $Y_{0}, \ldots, Y_{d}$ are in $L(D)$, the $R_{1}, \ldots, R_{k}$ will be in $L(r D)$ for some $r \geq 1$. In particular if we let $r$ be the largest degree of the $R_{i}$ 's in the $Y_{j}$ 's, then each $R_{i}$ will be in $L(r D)$. (Often this is true for a smaller $r$, in fact). 
Now let $\tau \in G$ be an automorphism of the code, and consider the image $\tau(\phi(X))$ of $\phi(X)$ under the induced action (6) on $\mathbb{P}^{d}$. The ideal of $\tau(\phi(X))$ is generated by $\tau\left(R_{1}\right), \ldots, \tau\left(R_{k}\right)$, by (5). If we can show that these functions are also in the ideal of $\phi(X)$, for any $\tau \in G$, then the ideals will be equal and we will have given an action of $G$ on $\phi(X)$, which we then pull back via $\phi$ to an action on $X$ : for $P \in X$,

$$
\tau(P)=\phi^{-1}(\tau(\phi(P))) .
$$

Let $R_{i} \in L(r D)$ be one of the minimal degree generators of the ideal of $\phi(X) ; R_{i}$ is of degree at most $r$ in $Y_{0}, \ldots Y_{d}$. Since the action of $G$ is linear on $L(D), \tau\left(R_{i}\right)$ will also be of degree at most $r$ in $Y_{0}, \ldots Y_{d}$, so $\tau\left(R_{i}\right)$ will also be in $L(r D)$. Since $R_{i}$ is in the ideal of $\phi(X), R_{i}$ vanishes at every point of $X$, including $P_{1}, \ldots, P_{n}$. Since as we showed above $\tau$ acts as a permutation of the points $P_{i}, \tau\left(R_{i}\right)$ must also vanish on $P_{1}, \ldots, P_{n}$, so $\tau\left(R_{i}\right)$ vanishes on $E$. This means that $\tau\left(R_{i}\right)$ is in $L(r D-E)$. But if $n>r \cdot \operatorname{deg}(D)$, then $r D-E$ is a divisor of degree $<0$ and $L(r D-E)$ is the trivial vector space, so $\tau\left(R_{i}\right)$ must vanish identically on $\phi(X)$. Thus $\tau\left(R_{i}\right)$ is in the vanishing ideal associated to $\phi(X)$, for each $R_{i}$ and for every $\tau \in G$.

We have shown that the action of $G$ on the code gives an action (6) on $\phi(X)$, which we then pull back via the embedding to an action on $X$. At each stage, the action was multiplicative, so we have a homomorphism $\psi: \operatorname{Perm} C \rightarrow \operatorname{Aut}(X)$. Using (7) it follows that $E$ is invariant under this action; we now need to show that the action leaves $D$ invariant. Consider an element $\tau$ of $G$ and its action on $D$. Because the action (8) of $\tau$ on $X$ was defined via an action (44) on $L(D)$, we know that $\tau$ preserves $L(D)$. But suppose that $\tau$ did not preserve $D$ itself, so that $\tau(D)=D^{\prime}, D \neq D^{\prime}$, but $L(D)=L\left(D^{\prime}\right)$. Then there must be a point $P$ in the support of $D$ such that its coefficient, $d_{P}$, in $D$ is larger than its coefficient $d_{P}^{\prime}$ in $D^{\prime}$. Now consider a function $f \in L(D)$. Because it is also in $L\left(D^{\prime}\right)$, we must have $\operatorname{div}(f)+D^{\prime} \geq 0$. Thus the coefficient of $\operatorname{div}(f)$ at $P$ must be at most $d_{P}^{\prime}$. Thus $\operatorname{div}(f)+D-\left(d_{P}-d_{P}^{\prime}\right) P \geq 0$, so in particular $f$ is in $L(D-P)$. This is true for any $f$ in $L(D)$, so $L(D)=L(D-P)$. But we assumed that $D$ was very ample; in particular $L(D)$ separates points, which means that $\operatorname{dim} L(D-P)=\operatorname{dim} L(D)-1$, a contradiction. So the action of $G$ on $X$ must preserve $D$. This means that the image of the homomorphism $\psi: \operatorname{Perm} C \rightarrow \operatorname{Aut}(X)$ is in $\operatorname{Aut}_{D, E}(X)$. 
It should be clear from these constructions that $\rho$ and $\phi$, when they exist, are inverses of each other, making $\operatorname{Aut}_{D, E}(X)$ and Perm $C$ isomorphic groups.

The result below is actually slightly stronger than the corresponding result of Wesemeyer (Corollary 4.9 [W] for elliptic curves and elliptic codes.

Corollary 3 Let $X$ be a smooth projective curve of genus $g \geq 2$. Let $D$ be a divisor on $X$ with $\operatorname{deg} D \geq 2 g+1$ and let $E$ be a collection of at least $(1+g) \operatorname{deg} D$ points on $X$ disjoint from the support of $D$. Then the group of permutation automorphisms of the code $C=C(D, E)$ is isomorphic to the group of automorphisms of $X$ that fix both $D$ and $E$.

proof: Since $\operatorname{deg} D \geq 2 g+1, D$ is very ample, so we use Theorem 2 want to estimate $r$. Suppose that the image of the embedding $|D|: X \hookrightarrow \mathbb{P}^{d}$ defined over $F$ is defined by multivariate polynomial relations $R_{1}=0, \ldots$, $R_{k}=0$ over $F$ of minimal degree. As noted in the proof of Theorem 2, we can take $r$ to be the maximal degree of the polynomials $R_{1}, \ldots, R_{k}$. Let $\bar{D}$ be the associated divisor over the algebraic closure $\bar{F}$. By "base-change", we see that the image of the associated embedding $|\bar{D}|: X \hookrightarrow \mathbb{P}^{d}$ defined over $\bar{F}$ is defined by the same multivariate polynomial relations $R_{1}=0, \ldots$, $R_{k}=0$ over $F$ (and hence over $\bar{F}$ ). Note that since $Y_{0}, \ldots, Y_{d}$ form a basis of $L(D)$, they are linearly independent, so $2 \leq r$ and $X$ cannot be contained in a hyperplane in $\mathbb{P}^{d}$.

If $d \geq 3$, Gruson, Lazarsfeld, and Peskine GLP (since $\bar{F}$ is algebraically closed) give the maximum degree of the $R_{i}^{\prime} s$ as $\operatorname{deg} D+1-d$ in most cases, or $\operatorname{deg} D+2-d$ if $X$ has genus zero and its image is smooth and has a $\operatorname{deg} D+2-d$-secant line. In our case, $d=\operatorname{dim} L(D)-1$ and $D$ is nonspecial, so $d=\operatorname{deg} D-g$. Therefore if $g \geq 2$, we will have $d \geq 3$ and from GLP], $r \leq 1+g$.

There a few special cases to consider that fall outside of Corollary 3. If $X$ is rational, and $d=1$, then the embedding is an isomorphism and the automorphism groups are the same. If $d=2$, then the embedding is as a plane conic, so $r=2$. For larger $d$, the theorem of GLP holds and shows that $r=2$ (and that $X$ always has a 2-secant line, which is not surprising). In both of these cases, the groups are isomorphic if $\operatorname{deg} E \geq 2 \operatorname{deg} D$. If $X$ has genus 1 and is embedded smoothly in $\mathbb{P}^{2}$, it must be as a cubic so $r=\operatorname{deg} D=3$; the groups will be isomorphic if $\operatorname{deg} E \geq 3 \operatorname{deg} D=9$. Again, for larger $d$ GLP] holds and shows that $r=2$, so the groups are isomorphic if $\operatorname{deg} E \geq 2 \operatorname{deg} D$. 
Remark 1 Under the hypotheses of Corollary 3, the length of $C$ is $n=$ $\operatorname{deg} E$, dimension is $k=\operatorname{deg} D+1-g$, and minimum distance $d \geq \operatorname{deg} E-$ $\operatorname{deg} D$ (see for example Corollary II.2.3 [Sti] $)$.

Example 4 Let $F=G F(49)$ and let $X$ denote the curve defined by

$$
y^{2}=x^{7}-x .
$$

This has genus 3 . The automorphism group $A u t_{F}(X)$ is a central 2-fold cover of $P G L_{2}(F)$ : we have a short exact sequence,

$$
1 \rightarrow H \rightarrow \operatorname{Aut}_{F}(X) \rightarrow P G L_{2}(7) \rightarrow 1,
$$

where $H$ denotes the subgroup of $A_{u} t_{F}(X)$ generated by the hyperelliptic involution (which happens to also be the center of $A_{u t}(X)$ ). For details, see [G], Theorem 1.

Next, we recall some consequences of $\S 3.2$ in [JT]. There are $|X(F)|=$ $2 \cdot 7^{2}-7+1=92$ F-rational points ${ }^{1}$ :

$$
X(F)=\left\{P_{1}=[1: 0: 1], P_{2}=[0: 0: 1], \ldots\right\} .
$$

The automorphism group does not act transitively on $X(F)$ but has 2 orbits: the orbit $C_{1}$ of $P_{1}$ and the orbit $C_{2}=X(F)-C_{1}$. We have $\left|C_{1}\right|=7+1=8$ and $\left|C_{2}\right|=2 \cdot 7 \cdot(7-1)=84$.

Let $D=m P_{1}, E=X(F)-C_{1}=\left\{Q_{1}, \ldots, Q_{84}\right\}$, and let

$$
C=C(D, E)=\left\{\left(f\left(Q_{1}\right), \ldots, f\left(Q_{84}\right)\right) \mid f \in L(D)\right\} .
$$

This is an $[n, k, d]$ code over $F$, where $n=\operatorname{deg}(E)=84, k \leq \operatorname{dim}(L(D))$.

Let $G=\operatorname{Stab}\left(P_{1}, A_{u t}(X)\right)$ denote the stabilizer of $P_{1}$. Since $E$ is an orbit of the full automorphism group, it will also be stabilized by $G$, so $G=$ $\operatorname{Aut}_{D, E}(X)$. The group $G$ is a non-abelian group of order $2 \cdot 7 \cdot(7-1)=84$.

According to Corollary 3. Perm $C(D, E)$ will be isomorphic to $G$ if we choose $m$ so that $\operatorname{deg} D$ is at least $2 g+1=7$ and $\operatorname{deg} E=84$ to be at least $(g+1) \operatorname{deg} D=4 \operatorname{deg} D$. Since $\operatorname{deg} D=m$, this means that $7 \leq m \leq 21$.

Assuming we choose $m>2 g-2=4$, the Riemann-Roch theorem implies $\operatorname{dim}(L(D))=m-2$, so $C$ is an $[84, m-2, \geq 84-m]$-code over $G F(49)$. Since $G$ fixes $D$ and preserves $E$, it acts on $C$ via

\footnotetext{
${ }^{1}$ MAGMA views the curve as embedded in a weighted projective space, with weights 1,4 , and 1 , in which the point at infinity is nonsingular.
} 


$$
g:\left(f\left(Q_{1}\right), \ldots, f\left(Q_{84}\right)\right) \longmapsto\left(f\left(g^{-1} Q_{1}\right), \ldots, f\left(g^{-1} Q_{84}\right)\right),
$$

for $g \in G$.

Remark 2 More generally, for $p>3$ and $p \equiv 3(\bmod 4)$, the curve $X$ defined ${ }^{2}$ by $y^{2}=x^{p}-x$ over $F=G F\left(p^{2}\right)$ is associated to an $[n, k, d]$ code $C$ over $F$, where $n=2 p(p-1), k=m-\frac{p-3}{2}, d \geq 2 p^{2}-2 p-m$, provided $m>p$. This code is the one-point $A G$ code constructed from the divisor $D=m P_{1}$, where $P_{1}=[1: 0: 1]$, and $E$ is the sum of the points in the orbit $X\left(G F\left(p^{2}\right)\right)-$ $X(G F(p))$ (see Proposition 3 of $[\mathrm{J}])$. When $m=p^{2}$, the parameters of this code beat the Gilbert-Varshamov bound [TV]. When $p<m<\frac{4 p(p-1)}{p+1}$, using the above corollary, it can be shown that the permutation automorphism group $P$ of $C$ is isomorphic to the stabilizer of $P_{1}$ in the automorphism group of $X$, which is of size $2 p(p-1)$. In this case, $P$ acts on $C$ as a subrepresentation of the regular representation. It would be interesting to know the decomposition of this representation.

In $[J]$, it is conjectured that $C$ has a permutation decoding algorithm of complexity $O(n)$. For a related discussion (for an $A G$ code constructed from a different curve), see [L].

In some interesting cases, there are not enough rational points on the curve to apply Theorem 2 .

Example 5 Again, let $X$ denote the genus 3 curve defined by

$$
y^{2}=x^{7}-x
$$

but this time over $F=G F(7)$. The automorphism group $A_{u} t_{F}(X)$ is now a central 2-fold cover of $P S L_{2}(F)$ : we have a short exact sequence,

$$
1 \rightarrow H \rightarrow \operatorname{Aut}_{F}(X) \rightarrow P L_{2}(7) \rightarrow 1,
$$

where as before $H$ denotes the subgroup of $A u t_{F}(X)$ generated by the hyperelliptic involution (which happens to also be the center of $\left.\operatorname{Aut}_{F}(X)\right)$. The

\footnotetext{
${ }^{2}$ This curve is embedded into weighted projective space, where $x$ and $z$ have weight 1 and $y$ has weight $\frac{p+1}{2}$.
} 
following transformations are generating elements of $G$ :

$$
\begin{gathered}
\gamma_{1}=\left\{\begin{array}{c}
x \longmapsto x, \\
y \longmapsto-y,
\end{array}, \quad \gamma_{2}=\gamma_{2}(a)=\left\{\begin{array}{c}
x \longmapsto a^{2} x, \\
y \longmapsto a y,
\end{array}\right.\right. \\
\gamma_{3}=\left\{\begin{array}{c}
x \longmapsto x+1, \\
y \longmapsto y,
\end{array}, \quad \gamma_{4}=\left\{\begin{array}{c}
x \longmapsto-1 / x, \\
y \longmapsto y / x^{4},
\end{array}\right.\right.
\end{gathered}
$$

where $a \in F^{\times}$is a primitive 6 - th root of unity.

On this curve there are only 8 F-rational points:

$X(F)=\left\{P_{1}=[1: 0: 0], P_{2}=[0: 0: 1], P_{3}=[1: 0: 1], \ldots, P_{8}=[6: 0: 1]\right\}$.

Thus it is impossible to choose $D$ and $E$ so that $\operatorname{deg} D \geq 7$ and $E$ consists of at least $4 \operatorname{deg} D$ distinct rational points. Let us instead choose $D=m P_{1}$ and $E$ to be all of the other rational points as before, and compare Perm $C$ and $\operatorname{Aut}_{D, E}(X)$.

The automorphism group acts transitively on $X(F)$; as in the previous example let $G=\operatorname{Aut}_{D, E}(X)=\operatorname{Stab}\left(P_{1}, A_{4}(X)\right)$, the stabilizer of the point at infinity in $X(F)$. (All of the stabilizers $\operatorname{Stab}\left(P_{i}\right.$, Aut $_{F}(X)$ ) are conjugate to each other in $\left.A_{u t}(X), 1 \leq i \leq 8\right)$. The group $G$ is a non-abelian group of order 42 (In fact, the group $G / Z(G)$ is the non-abelian group of order 21 , where $Z(G)$ denotes the center of $G$.) Take the automorphisms $\gamma_{1}, \gamma_{2}$ with $a=2$ and $\gamma_{3}$ as generators of $G$. If we identify $S=\left\{P_{2}, \ldots, P_{8}\right\}$ with $\{1,2, \ldots, 7\}$ then

$$
\begin{gathered}
\gamma_{1} \leftrightarrow(2,7)(3,6)(4,5)=g_{1}, \\
\gamma_{2} \leftrightarrow(2,5,3)(4,6,7)=g_{2}, \\
\gamma_{3} \leftrightarrow(1,2, \ldots 7)=g_{3} .
\end{gathered}
$$

Let $D=5 P_{1}, S=C(F)-\left\{P_{1}\right\}$, and let

$$
C(D, E)=\left\{\left(f\left(P_{2}\right), \ldots, f\left(P_{8}\right)\right) \mid f \in L(D)\right\} .
$$

This is a $[7,3,5]$ code over $F$. In fact, $\operatorname{dim}(L(D))=3$, so the evaluation map $f \longmapsto\left(f\left(P_{2}\right), \ldots, f\left(P_{8}\right)\right), f \in L(D)$, is injective. Since $G$ fixes $D$ and preserves $E$, it acts on $C$ via

$$
g:\left(f\left(P_{2}\right), \ldots, f\left(P_{8}\right)\right) \longmapsto\left(f\left(g^{-1} P_{2}\right), \ldots, f\left(g^{-1} P_{8}\right)\right),
$$


for $g \in G$.

Let $P$ denote the permutation group of this code. It a group of order 42. However, it is not isomorphic to $G$ ! In fact, $P$ has trivial center. The (permutation) action of $G$ on this code implies that there is a homomorphism

$$
\rho: G \rightarrow P .
$$

What is the kernel of this map? There are two possibilities: either a subgroup of order 6 or a subgroup of order 21 (this is obtained using [GAP] by matching possible orders of quotients $G / N$ with possible orders of subgroups of $P$ ). Indeed, the kernel ker $(\phi)=N=\left\langle g_{2}, g_{3}\right\rangle$ is a non-abelian normal subgroup of $G=\left\langle g_{1}, g_{2}, g_{3}\right\rangle$ of order 21 .

\section{Acknowledgements}

We thank Jessica Sidman for the reference in the proof of Corollary 3 and Will Traves for many helpful conversations.

\section{References}

[GAP] The GAP Group, GAP - Groups, Algorithms, and Programming, Version 4.3; 2002, http://www.gap-system.org.

[G] V. D. Goppa, Geometry and codes, Kluwer, 1988.

[GLP] L. Gruson, R. Lazarsfeld, and C. Peskine, "On a Theorem of Castelnuovo, and the equations defining space curves," Invent. Math. 72, 491506 (1983).

[GUAVA] GAP error-correcting codes package GUAVA http: //cadigweb.ew. usna.edu/ ${ }^{\sim}$ wdj/gap/GUAVA/

[HLS] C. Heegard, J. Little, K. Saints, "Systematic encoding via Gröbner bases for a class of algebraic-geometric Goppa codes," IEEE Trans. Infor. Theory $\underline{41}(1995) 1752-1761$

[J] Joyner, D. "Conjectural permutation decoding of some AG codes," to appear, SIGSAM Bull. 
[JK] - and A. Ksir, "Decomposing representations of finite groups on Riemann-Roch spaces," preprint 2003, available at http://front.math.ucdavis. edu/math. AG/0312383

[JT] - and W. Traves, "Representations of finite groups on Riemann-Roch spaces," preprint, 2002 available at http://front.math.ucdavis.edu/math.AG/0210408 (revised version posted April, 2004)

[L] J. Little, "The algebraic structure of some AG Goppa codes," in Proceedings of the 33rd Annual Allerton Conference on Communication, Control, and Computing, October 4-6, 1995.

[MAGMA] W. Bosma, J. Cannon, C. Playoust, "The MAGMA algebra system, I: The user language," J. Symb. Comp., 244(1997)235-265. (See also the MAGMA homepage at http://www . maths . usyd.edu .au:8000/u/magma/).

[Sti] H. Stichtenoth, Algebraic function fields and codes, SpringerVerlag, 1993.

[TV] M.A. Tsfasman and S.G. Vlädut, Algebraic-geometric codes, Kluwer Academic Publishers, 1991.

[W] S. Wesemeyer, "On the automorphism group of various Goppa codes," IEEE Trans. Info. Theory., 44(1998)630-643. 